\title{
NUCLEUS GRACILIS RESPONSES TO KNEE JOINT MOTION: A FRE- QUENCY RESPONSE STUDY
}

\author{
W. J. WILliAMS, S. L. BeMENT, T. C. T. YIN AND W. D. MCCALL, Jr.
}

Bioelectrical Sciences Laboratory, Department of Electrical and Computer Engineering and the Bioengineering Program, University of Michigan, Ann Arbor, Mich. 48105 (U.S.A.)

(Accepted June 8th, 1973)

SUMMARY

(1) A systems analysis approach was used to study the dynamic responses of sensory cells in the nucleus gracilis of anesthetized cats. Sinusoidal changes in knee angle were used as inputs and the average firing rate of single gracile cells was recorded as the output. Frequency response functions were derived from data displayed as Bode plots.

(2) Fifty-nine cells were studied. The majority of these cells exhibited an acceleration sensitivity but 5 cells exhibited a velocity sensitivity. No position sensitivity was evident and no slowly adapting or tonic responses were recorded.

(3) The acceleration sensitive cells demonstrated significant non-linear responses. The gain associated with their frequency response function depended upon both static knee angle and input excursion amplitude. These cells also exhibited a bi-directional response to sinusoidal and square wave inputs.

(4) These data are taken as evidence that the dorsal columns may carry acceleration and velocity information from receptors in the knee joint, but that positional information may travel by other pathways to cells located in other areas. If such is the case, lesion experiments involving the dorsal columns should reveal loss of velocity and acceleration sense but no decrement in position sense.

INTRODUCTION

Physiologists have long known that humans are able to judge accurately the position and movement of their joints without the aid of visual or tactile cues 21 . This sense is thought to be relayed by receptors that lie deep within the joints rather than in the muscle ${ }^{6,28,35}$. These joint receptors, most frequently investigated in the knee of the cat, arise from innervation in and around the joint capsule14-16,36. The 
responses of the slowly adapting receptors to both static and dynamic stimulation are well-known $1,4,8,10,11,13,26,27,36$.

Although the characteristics of the peripheral receptors have been the subject of several investigators, the properties of their central projections are not well understood. In fact, the relevant central pathways themselves are not well known. The traditional view was that all fibers from deep receptors in the knee joint ascended in the dorsal columns to synapse in the nucleus gracilis ${ }^{12,19}$. However, this concept has come under attack recently on the basis of both electrophysiological and psychophysical evidence $7,10,17,23,32,37-40$. The projections of the numerous slowly adapting joint receptors have been reported to leave the dorsal columns by $C_{3}$ and only the few rapidly adapting fibers project to the gracile nucleus 7,10 . The pathways for the slowly adapting fibers to higher levels are not known.

The only quantitative study of central joint neurons was reported by Mountcastle et al. ${ }^{31}$ in the ventrobasal thalamus of unanesthetized monkeys. They studied the response of these supposed third order cells to static position of the joint. Electrophysiological studies of the second order neurons in the gracile nucleus have acknowledged the presence of cells sensitive to joint stimulation but have not attempted any quantitative analysis 24,43 .

In recent years a growing number of investigators have begun to use systems theory to study the response of various biological receptors in an effort to quantify the response to dynamic stimulation. The response dynamics for muscle spindles ${ }^{22,25,34}$, crustacean stretch receptors ${ }^{5}$, and other mechanoreceptors ${ }^{9,26}$ have been studied. For all of these mechanoreceptors the frequency response can be characterized as a high pass filter, i.e., the receptor shows a greater sensitivity to high frequency input signals than to low frequency signals. Presumably this provides the central nervous system with increased capability for responding to rapidly changing stimuli.

The purpose of our research was to describe the properties of knee joint afferent responses recorded in the gracile nucleus of the cat. We have used dynamic stimulation and systems analysis techniques to characterize the properties of these second order cells. The results reported here are part of a long-term study of the joint receptor system that includes responses at the receptor level and thalamic level. Preliminary results obtained at the knee joint, gracile, and thalamic levels have been reported $^{26,27,41,44}$.

METHODS

Surgery

A total of 36 cats were used in this study. They were initially anesthetized by intraperitoneal injection of sodium pentobarbital $(35 \mathrm{mg} / \mathrm{kg})$. A cannula was placed in the cephalic vein, and supplementary doses of physiological saline and anesthetic were given in order to maintain a light analgesic state. The rectal temperature was monitored and maintained at $36-38^{\circ} \mathrm{C}$. The left hind leg was skinned and denervated except for the medial and posterior articular nerves from the knee joint capsule. The femur was firmly pinned and the lower leg connected to a mechanical fixture so 
that the knee joint could be rotated through a controlled angle. A potentiometer positioned at the joint axis monitored the knee joint angle.

The animal was placed in a standard stereotaxic apparatus. The head was arched forward with respect to the body to facilitate access to the nucleus gracilis and also to lessen the effects of respiratory movements during the recording sessions. After a wide bilateral laminectomy at the level of the obex, a tungsten microelectrode was advanced into the nucleus gracilis. Standard techniques for extracellular recording from single units were employed.

The nucleus gracilis was probed systematically for cells that responded to movement or position of the knee joint, but not to cutaneous stimulation of any surface area. The knee joint area and the involved hind limb, tail, and rear part of the body were tapped, pressed, and manipulated to establish that the gracile response originated in the knee joint. Even with the extensive denervation procedure, a number of responses from receptive fields within the denervated area were observed. Usually only a few knee joint units could be positively identified during a given experiment. These cells always exhibited sensitivity to the following tests: joint movement within some active angle, pressure applied to the joint capsule, and twisting of the tibia. There was no response to light cutaneous stimuli applied around the hind limb. Once a joint response was positively determined, the response type and sensitivity from flexion to extension were noted. The limb was moved by hand in this case and qualitative judgments were made under these conditions.

\section{Application of experimental stimuli}

Once the range of response of the unit was determined, the mechanical fixture was attached to the limb and the precise quantitative knee joint movements were applied by a voltage controlled electromechanical actuator ${ }^{18}$. The last 9 experiments were performed using an improved rotational version of the device, allowing larger excursions of angle. Discrete sine, ramp and step inputs were applied in the frequency range of $0.01-7 \mathrm{~Hz}$. In order to obtain frequency response profiles we also applied a logarithmically swept sine wave of joint angle over a frequency range of $0.1-7 \mathrm{~Hz}$, as obtained from a Hewlett-Packard 3300A function generator with plug-in module 3305 .

The ideal situation was to determine the response profiles under 6 different conditions. These 6 conditions consisted of separate frequency response profiles at 3

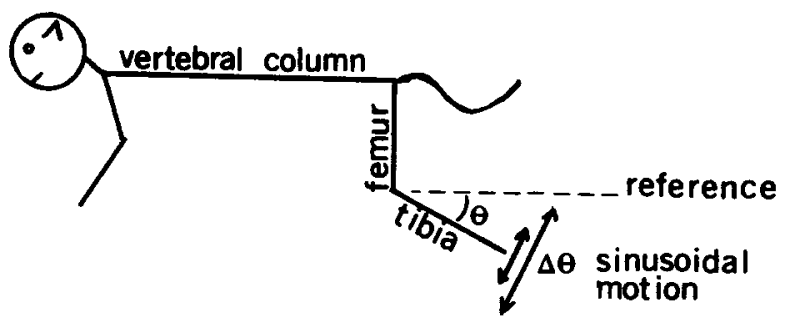

Fig. 1. Definition of $\theta$ and $\Delta \theta$. The stick figure indicates that $\theta$ is the static bias angle referenced to the horizontal axis shown and that $\Delta \theta$ is size of the peak-to-peak sinusoidal excursion about $\theta$. 
bias angles, $\theta$, within the response range of the unit while maintaining the angular excursion $\Delta \theta$ of the sinusoidal motion at a constant value (see Fig. 1 for a clarification of $\theta$ and $\Delta \theta$ ). Three additional frequency response profiles at a constant $\theta$ with 3 values of the excursion $\Delta \theta$ completed the set of 6 profiles. Since each frequency response determination required a minimum of $500 \mathrm{sec}$, at least $3000 \mathrm{sec}$ or $50 \mathrm{~min}$ plus the time required to change the $\theta$ or $\Delta \theta$ settings between the 6 tests were needed to complete all the frequency response runs. Often the unit would be lost before completing the entire set of discrete signals and frequency response profiles. The vibrations induced in moving the leg, particularly at the higher frequencies, often caused the loss of the single unit under study at some point in the sequences of experiments.

\section{Data acquisition and processing}

The amplified and filtered $(80 \mathrm{~Hz}-5 \mathrm{kHz})$ single unit impulses and the voltage proportional to instantaneous knee joint angle were recorded on magnetic tape for later computer analysis. During computer processing a window amplitude discriminator was used to convert the action potentials into pulses of standard height and duration. Knee joint angle was taken to be the input parameter and the time average spike rate of a single responding cell was used as the system output. Cycle histograms were computed from the single sinusoid data. Fourier series analyses were performed on these results to obtain the fundamental and higher harmonic components.

The data acquisition was improved markedly by a novel technique wherein the input was swept over a frequency range $0.1-7 \mathrm{~Hz}$. This technique enhanced our ability to obtain frequency response profiles of a single cell in a relatively short time. A plot of knee angle versus time (the swept frequency input) is shown in the upper left of Fig. 2. Computer implemented correlation and Fourier transform techniques were used to process the data and to extract the system frequency response (Bode plot). A summary diagram of the data processing technique is given in Fig. 2. Only the fundamental component of the output average neural firing rate was used to obtain the frequency response profile, the so-called describing function approach. Any dynamic effects inherent in the instrumentation were removed by the computation procedure so that the transfer function obtained represented only the transfer function of the biological system. The results were displayed as Bode plots ${ }^{29}$. A detailed description of this technique is available ${ }^{42}$.

\section{RESULTS}

One of the most striking features we observed in searching the gracile nucleus was the difficulty in finding knee joint sensitive cells. Even with the extensive denervation procedure, the number of cells responsive solely to joint movement or position was still a small percentage of the total number of active units isolated.

Out of the several hundred gracile responses observed, 59 were considered to be positively identified knee joint units and qualitative observations were made on these cells. In 47 cases it was possible to complete some of the different experimental paradigms. In 35 cases we were able to at least complete one frequency response 


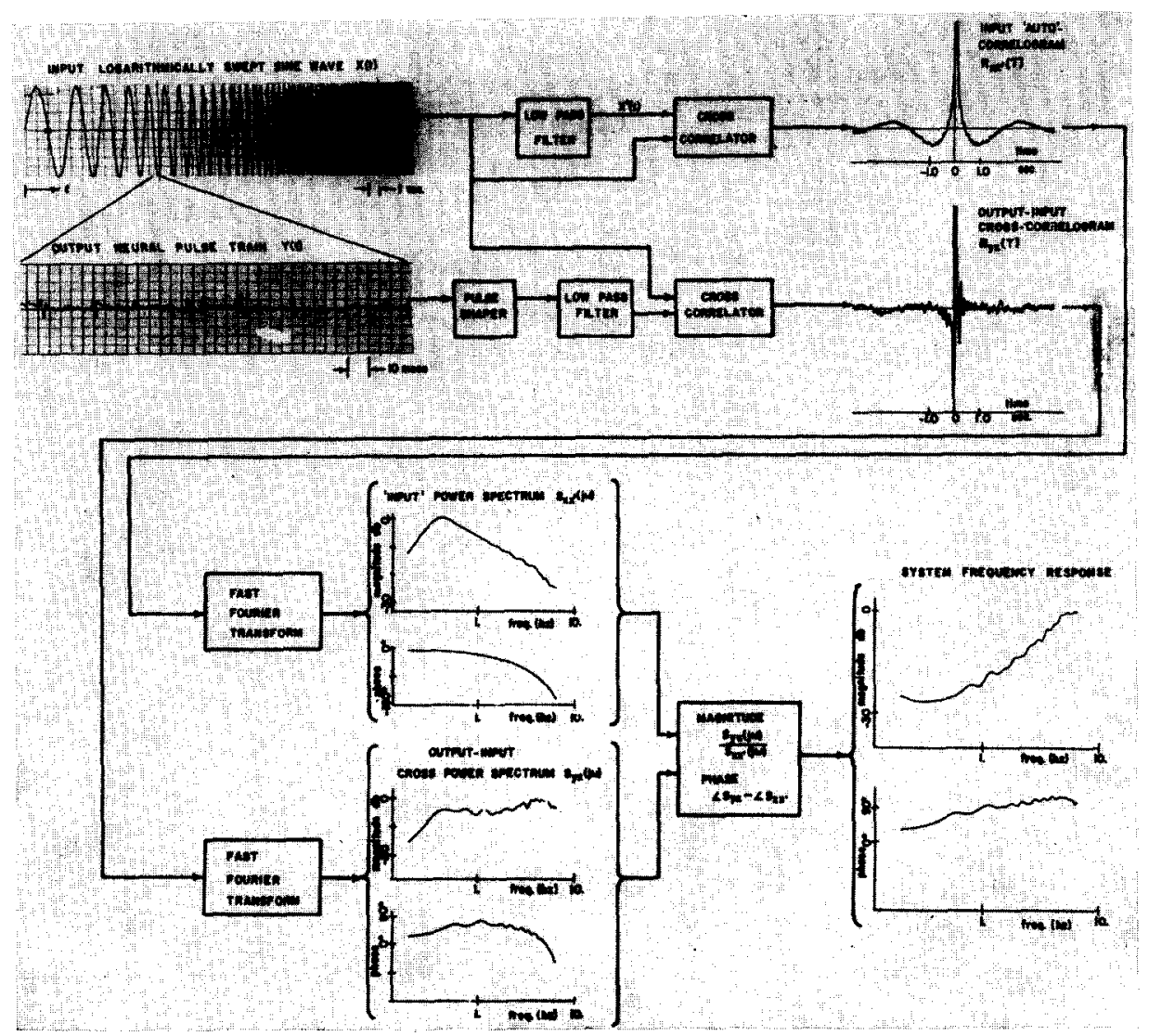

Fig. 2. Transfer function extraction technique. This diagram summarizes the steps in the data progressing sequence. The graphs and waveforms shown are samples of typical gracile data. The neural spike train is shaped into standard $1 \mathrm{msec}, 10 \mathrm{~V}$ pulses by a window discriminator. The low pass filter then 'demodulates' the pulse train. In order to cancel the dynamic filtering effects of the filter the input signal is passed through an identical filter so that the same effect appears in both Sxx' and Syx. Sxx' is therefore Sxx modified by the filter characteristic. Rxx' and Sxx' are not truly $\mathbf{R x x}$ and Sxx, but filter modified versions of these quantities. Further details are given in Williams et al. ${ }^{42}$.

determination. In only 4 cases were we able to complete the entire set of 6 frequency response profiles. Our conclusions with regard to the frequency response of individual units are based upon the 35 cells in which a determination of the response was made under 1-6 experimental conditions. Our conclusions with regard to the influence of bias angle and amplitude on the frequency response are, of course, based primarily upon the 4 cells in which fortuitous recording conditions permitted a comprehensive study of response profile. Inferences drawn from these 4 cells must be viewed with caution due to the limited sample size. However, there is no question of reliability of the 'incomplete' data sets. We were just not able to meet all of the desired and self-imposed criteria for a 'complete' data set. Thus the 'incomplete' data sets augment the basic observations made concerning the 'complete' data sets.

By far the most common type of joint cell (54 of 59) encountered had rapidly adapting or phasic characteristics, i.e., it responded with a short burst of spikes only 

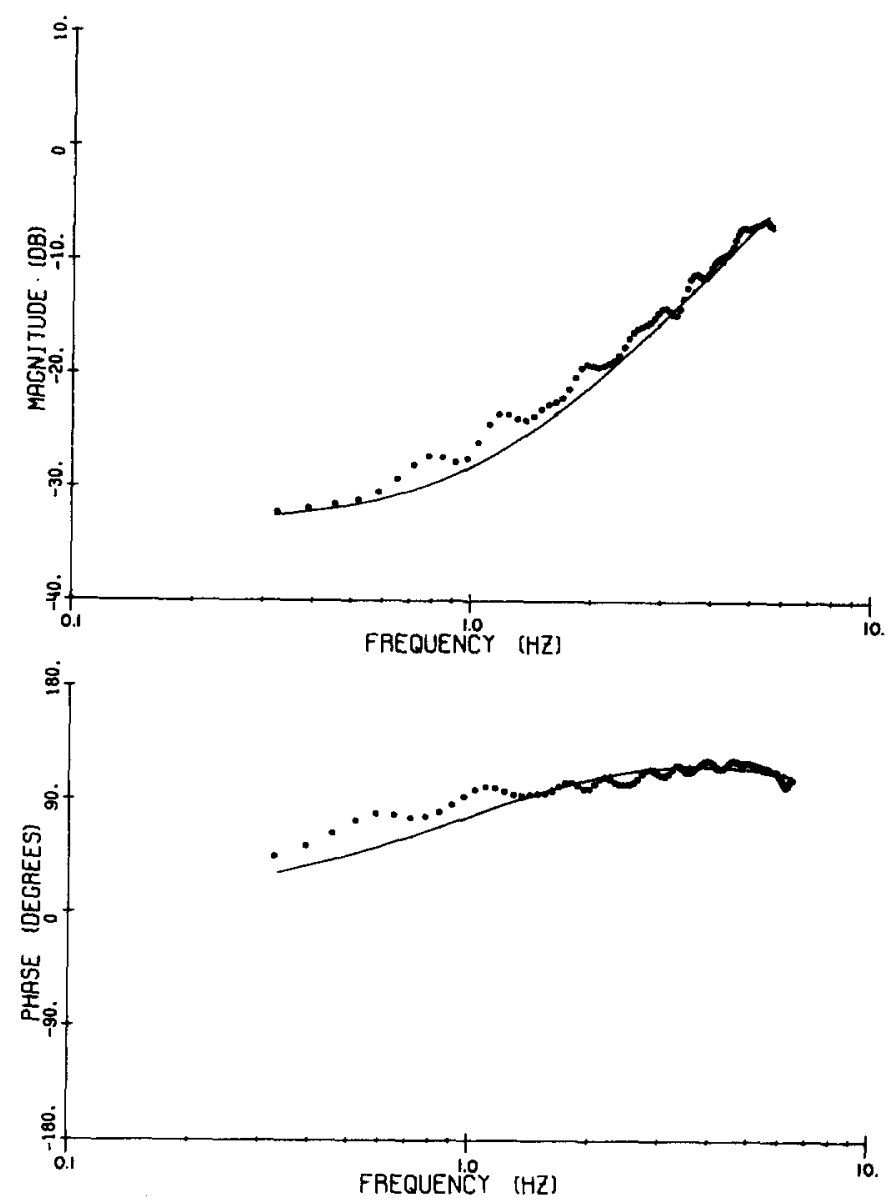

Fig. 3. Frequency response of a typical gracile cell. This cell exhibited the typical acceleration sensitive response of the majority of cells studied at the gracile level. The model equation (solid line) was fitted to the experimental data by use of a least-mean-square-error fitting program. The shape of the frequency response was relatively invariant for different $\theta$ and $\Delta \theta$. Zero $\mathrm{dB}$ is equivalent to 8.5 pulses/sec/degree.

when the joint was moved and did not respond when the limb was held steady. These cells responded over a broad range of knee angle, and exhibited rapid, irregular adaptation to step changes in knee angle. We shall call these type I, rapidly adapting receptors. The other type of joint cell found ( 5 of 59) were also phasic in the sense that they responded only to changes in joint angle. However, these cells responded with a slower rate of adaptation to step inputs and had a steadier firing rate when stimulated. However, they always adapted to a zero firing rate after several seconds if the limb was held in a fixed position and they responded only when the limb was positioned near the limits of flexion or extension. These cells we call type II, rapidly adapting.

In our extensive search of the gracile nucleus, no slowly adapting or tonic cells sensitive to knee joint position similar to those commonly seen at the peripheral nerve level were found. Neither the type I nor type II rapidly adapting cell types were capable of signaling static joint position. 
The differences in the type I and type II receptors were also clearly manifested in their dynamic responses. The frequency response profile of a typical type $I$ cell is shown in Fig. 3. The dots represent the experimental frequency response data extracted by the computer analysis. The slope of the Bode plot magnitude curve is typically about $40 \mathrm{~dB}$ /decade from 1 to $7 \mathrm{~Hz}$ and suggests a second derivative or acceleration response over that frequency range (see Appendix). The solid line represents a least squares fit of a model for the data, extracted by a conjugate gradient optimization approach. The model form is given by: $H(s)=\left\{G(s+z)^{2} /(s+p)\right\} e^{-s \tau}$.

The optimized parameters of the model indicate a double zero at $1 \mathrm{~Hz}(\mathrm{z}=2 \pi)$, a pole at $15 \mathrm{~Hz}(\mathrm{p}=30 \pi)$, and a pure time delay $(\tau)$ of $13 \mathrm{msec}$. The $13 \mathrm{msec}$ pure time delay is consistent with experimental response latencies of $12-15 \mathrm{msec}$ recorded when step inputs of knee angle were applied. Both the experimental data and the model demonstrate second derivative properties over the frequency range of $1-7 \mathrm{~Hz}$, as indicated by the $40 \mathrm{~dB} /$ decade magnitude slope in that region. Therefore, the response of the type I rapidly adapting gracile cells to dynamic changes in knee angle appears to be principally the second derivative of position, i.e., acceleration.

The type II rapidly adapting receptors exhibited a significantly different type of frequency response, as shown in Fig. 5. Their frequency response profile was more consistent with a $20 \mathrm{~dB} /$ decade magnitude slope and a $90^{\circ}$ phase lead model, which suggests a first derivative or velocity sensitive frequency response. The solid line in Fig. 5 represents an optimized model fit to the experimental data points. The transfer

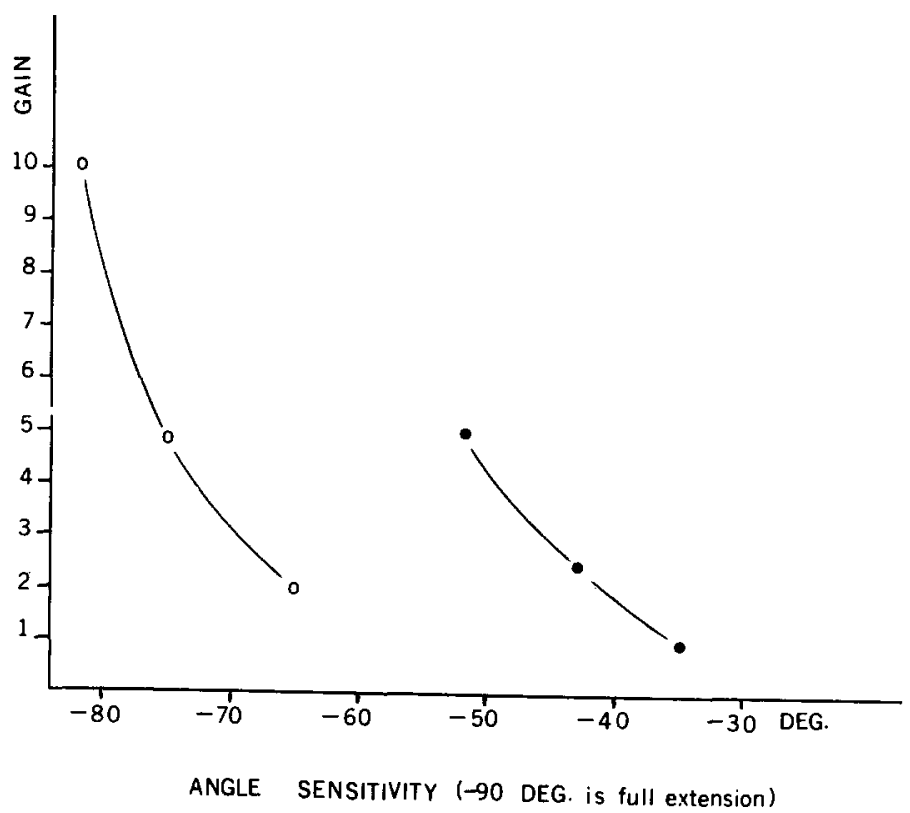

Fig. 4. Effect of joint bias angle on the response of 'acceleration sensitive' gracile cells. Closed circles: unit $25, \Delta \theta=9^{\circ}$; open circles: unit $29, \Delta \theta=12^{\circ}$. The gain decreases as the leg is biased toward flexion in this case. Each of the data points represents the gain $\mathrm{G}$ extracted from a single complete frequency response such as shown in Fig. 3. 

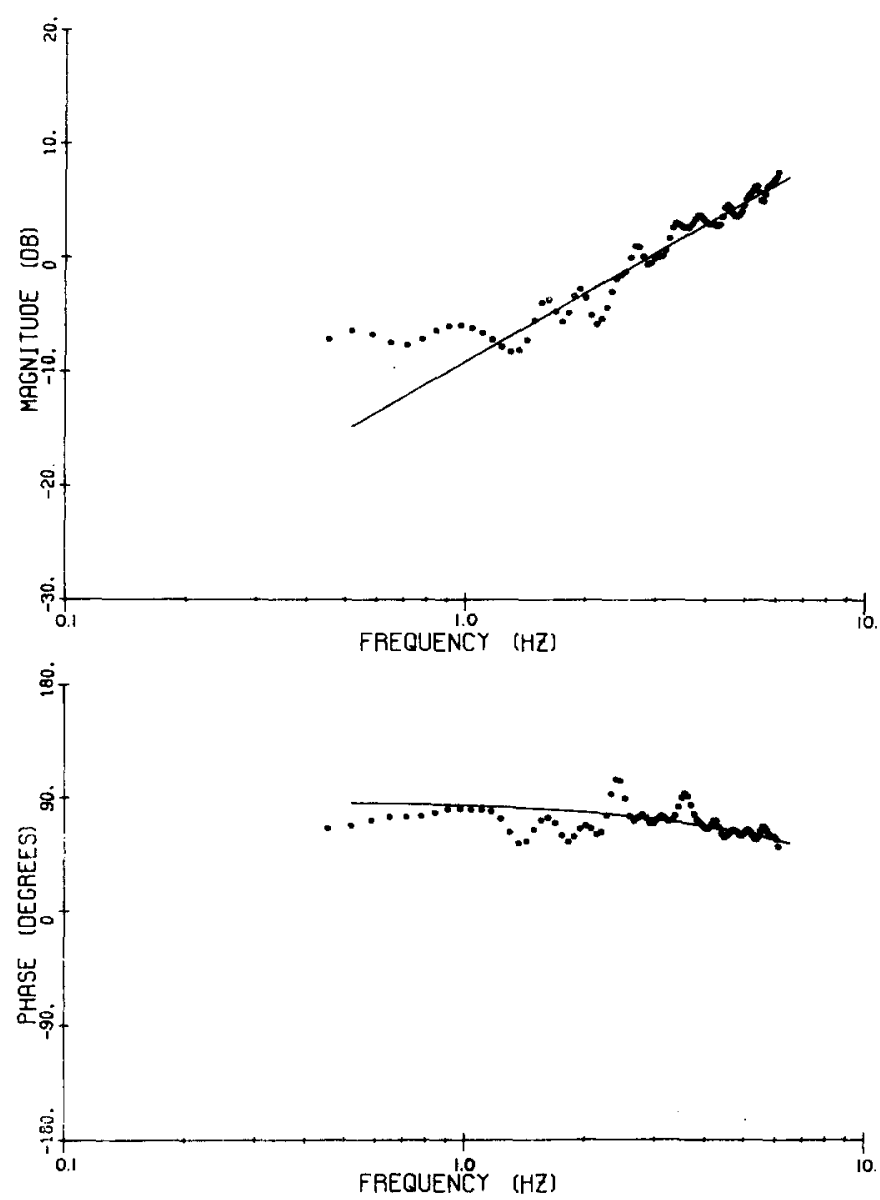

Fig. 5. Velocity sensitive response. The frequency response profile of a 'velocity sensitive' cell is shown. The solid line represents a model equation of the form $\mathrm{G} \cdot \mathrm{s} \cdot \mathrm{e}^{-\mathrm{s} \tau}$ and the symbols represent the experimental data. The model equation was fitted to the data by the least squares program. The slope of the magnitude portion of the response is approximately $20 \mathrm{~dB} /$ decade above $1 \mathrm{~Hz}$, indicating velocity sensitivity (as predicted by the model equation). The time delay $\tau$ required to fit the phase portion of the response (in addition to the $90^{\circ}$ phase lead of the differentiater operator) is $16 \mathrm{msec}$, in good agreement with step response data. Zero $\mathrm{dB}$ is equivalent to $1.0 \mathrm{pulses} / \mathrm{sec} / \mathrm{degree}$.

function of this model is expressed by an equation of the form $H(s)=G \cdot s \cdot e^{-s \tau}$, where $\tau$ is $16 \mathrm{msec}$.

The linearity of the system to sinusoidal inputs was tested in two ways: (1) at a constant bias angle $\theta$, the frequency response curves were obtained for different excursion angles, $\Delta \theta$; and (2) at a constant excursion angle $\Delta \theta$, the frequency response curves were obtained for different bias angles $\theta$. In general, the shape of the Bode plot for a particular cell was unchanged as either $\theta$ or $\Delta \theta$ was varied. However, the steady state gain of the response was found to be a function of bias angle and excursion, i.e., the entire curve was displaced. In the models this is represented by the parameter G. Fig. 4 shows the type I units in which the gain increased as the bias angle shifted toward full extension. Each of the data points represents the gain 

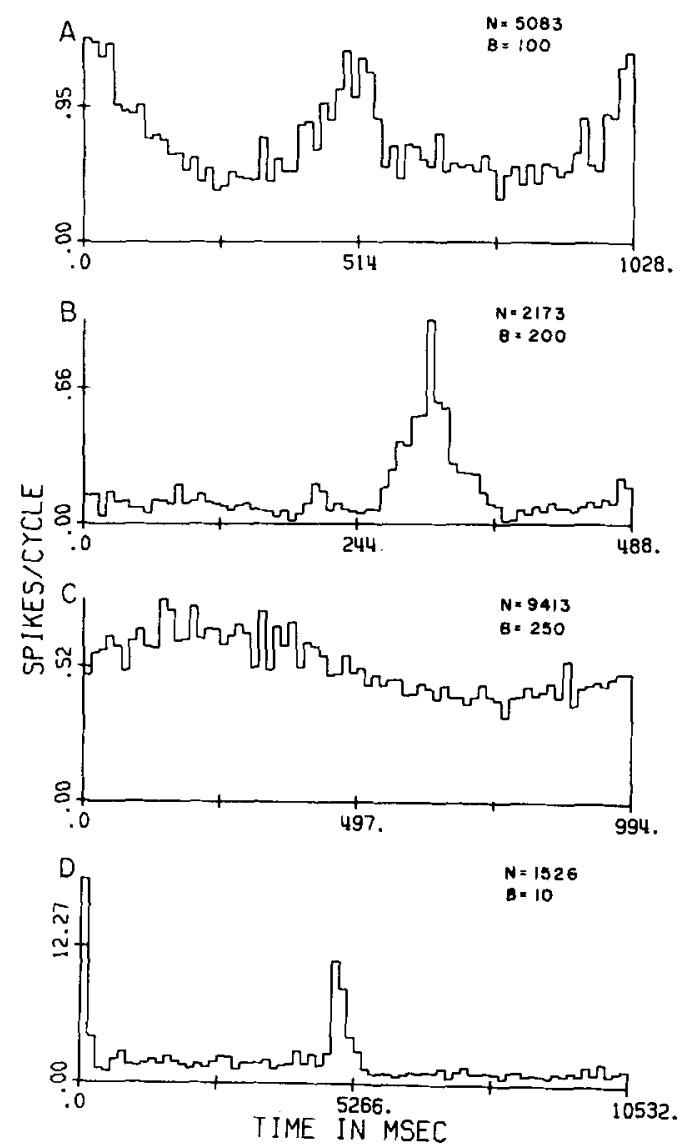

Fig. 6. Cycle histograms for gracile cell responses. These histograms represent the number of times a cell fired during a particular time interval, where the total time (horizontal axis) is the time per cycle of a constant frequency input. $\mathbf{B}$ is the number of cycles analyzed and $\mathbf{N}$ is the total number of spikes represented in the histogram. Histograms $A-C$ are for a sinusoidal input and $D$ is for a square wave input. The bi-directional response characteristic of acceleration sensitive gracile cells is shown in A. Histogram B indicates the uni-directional phase-locked response characteristic found in many acceleration sensitive cells at higher stimulus frequencies. Histogram $C$ is a velocity sensitive cell. Histogram D represents the response of an acceleration sensitive cell to a square wave input.

G extracted from a single complete frequency response such as shown in Fig. 3 and optimized by the least squares conjugate gradient search. We also observed cells that showed increased gain as the bias angle shifted toward flexion. The gain $G$ also increased with increased excursion $\Delta \theta$. If the system was linear, the gain should be independent of the input bias angle and amplitude. However the gain of the system at the gracile level appears to be a function of both bias angle and excursion.

Cycle histograms were computed for the response of gracile cells to discrete sine and square wave inputs. Typical responses of the type I and type II cells are shown in Fig. 6. Many type I units responded with a bi-directional characteristic, as indicated by the two distinct peaks in the cycle histogram of Fig. 6A. Other type I cells responded with a uni-directional phase-locked component represented by a 
single peak in the cycle histogram of Fig. 6B. Such phase-locking was particularly evident at higher frequencies. The bi-directional and phase-locking characteristics indicate non-linearities that are not predicted by transfer functions derived from the Bode plot results.

The response of a type II gracile cell to a sinusoidal input waveform is shown in Fig. 6C. The cycle histogram reflects the sinusoidal shape of the input waveform more faithfully than is the case for acceleration sensitive responses. This ensues from the steadier firing rate of these cells.

The response of a type I cell to a square wave input waveform of joint angle is shown in Fig. 6D. The initial burst of spikes following a step change in knee angle had a latency of $12-15 \mathrm{msec}$ in both type I and the type II responses.

All of the units identified as joint neurons were found along the medial aspect of the gracile nucleus. In our initial efforts we explored the nucleus in a uniform manner; but when it became clear that the hind limb cells were located medially, we biased our sample to the midline area in order to enhance the possibilities of finding a knee joint cell. Most of the joint cells were located near the level of the obex or caudal to it. Since our primary purpose was to examine the dynamic response characteristics of gracile cells that responded to knee joint motion, we did not attempt to establish a careful somatotopic mapping of the knee joint to the gracile nucleus.

Table I summarizes the basic results obtained in the two types of cell populations.

TABLE I

SUMMARY OF JOINT MOTION RESPONSES

\begin{tabular}{|c|c|c|c|}
\hline & Slowly adapting & Rapidly adapting $I I$ & Rapidly adapting I \\
\hline $\begin{array}{l}\text { System } \\
\text { characterization }\end{array}$ & $\begin{array}{l}\text { Quarter order } \\
\text { differentiator } \\
(0.01-7 \mathrm{~Hz})\end{array}$ & $\begin{array}{l}\text { Differentiator } \\
(1-7 \mathrm{~Hz})\end{array}$ & $\begin{array}{l}\text { Double differentiator } \\
(1-7 \mathrm{~Hz})\end{array}$ \\
\hline Proposed sensor type & Position & Velocity & Acceleration \\
\hline Directional sensitivity & $\begin{array}{l}\text { Increased firing with } \\
\text { flexion and decreased } \\
\text { firing with extension } \\
\text { or vice versa } \\
\text { (uni-directional) }\end{array}$ & $\begin{array}{l}\text { Same as slowly } \\
\text { adapting }\end{array}$ & $\begin{array}{l}\text { Firing increase with } \\
\text { both flexion and } \\
\text { extension } \\
\text { (bi-directional or } \\
\text { uni-directional) }\end{array}$ \\
\hline $\begin{array}{l}\text { Adaptation to step } \\
\text { change position }\end{array}$ & $\begin{array}{l}\text { Regular decline to } \\
\text { steady firing rate }\end{array}$ & $\begin{array}{l}\text { Regular decline to zero } \\
\text { firing rate }\end{array}$ & $\begin{array}{l}\text { Irregular decline to } \\
\text { zero firing rate }\end{array}$ \\
\hline $\begin{array}{l}\text { Projections to gracile } \\
\text { nucleus }\end{array}$ & Few, if any ( 0 of 59$)$ & Few (5 of 59 ) & Many (54 of 59) \\
\hline $\begin{array}{l}\text { Activation sensitivity of } \\
\text { gracile responses }\end{array}$ & & $\begin{array}{l}\text { Responds only near } \\
\text { limits of flexion and } \\
\text { extension, over a } \\
\text { narrow range of angle }\end{array}$ & $\begin{array}{l}\text { Responds over broad } \\
\text { range of knee angle, } \\
\text { response increases } \\
\text { toward extreme of } \\
\text { either flexion or } \\
\text { extension }\end{array}$ \\
\hline
\end{tabular}


DISCUSSION

The absence of any slowly adapting responses at the gracile level conflicts with the classical concept that these units ascend to the dorsal column nuclei. Burgess and Clark $^{7,10}$ report similar findings based upon other electrophysiological techniques. In addition, recent psychophysical experiments indicate that joint position sense reaches somatosensory cortex via pathways other than the dorsal columns ${ }^{37}$. Suggestions have been made that slowly adapting afferents ascend in the dorsal lateral tracts $17,32,40$.

On the other hand, slowly adapting responses have been reported in the dorsal column nuclei ${ }^{24}$, the somatosensory thalamus ${ }^{31,33}$, and cortex ${ }^{30}$. However, some investigators apparently do not interpret the term 'slowly adapting' as we do. The slowly adapting response reported at the gracile level ${ }^{24}$ were recorded with the limb in motion and appear to be similar to the type II responses we observed. We found the rate of adaptation of the type II gracile cells to be less than the rate of adaptation of the type I cells, but the type II cells always adapted to a zero rate of discharge if the limb was held in a fixed position for several seconds. In contrast, we have found receptors that we term 'slowly adapting' at the knee joint level ${ }^{26,27}$. These receptors adapt to a relatively steady rate of discharge and hold this discharge rate for long periods. The type II responses and slowly adapting responses do share a common feature in that the response to step inputs adapts at a steady rate, whereas the type I response adapts rapidly and irregularly.

The slope of the magnitude portion of the transfer functions of the slowly adapting receptors is in the order of $5 \mathrm{~dB} /$ decade $^{26,27}$. The frequency response of a slowly adapting receptor at the knee joint level is compared with the transfer function of an acceleration sensitive gracile cell in Fig. 7. The Bode plots of these two cells are distinctively different. The gracile results never yielded Bode plots with magnitude slopes on the order of $5 \mathrm{~dB} /$ decade, as is commonly observed from the slowly adapting receptor data at the knee joint level. Any gracile level responses that might be called 'slowly adapting' are associated with Bode plots with magnitude slopes in the order of $20 \mathrm{~dB} /$ decade beyond $1 \mathrm{~Hz}$, again markedly different from the Bode plot results for the 'slowly adapting' receptors studied at the knee joint level.

The broad terms 'slowly adapting' and 'rapidly adapting' often serve to create confusion since each investigator uses his own interpretation in describing research results. A definitive description of receptor response such as represented by a Bode plot may be more useful in identifying the several response types.

\section{Surgical and anesthetic effects}

Surgical and anesthetic effects are more pronounced in a study of the temporal response characteristics of neurons than in an anatomical or somatotopical study ${ }^{33}$. Therefore, attention should be given to the effects of denervation and anesthesia. The extensive denervation procedure may alter the normal loading on the joint and thereby modify the response of the joint receptors. However, without the denervation procedure locating and isolating the relatively rare joint response among the many 

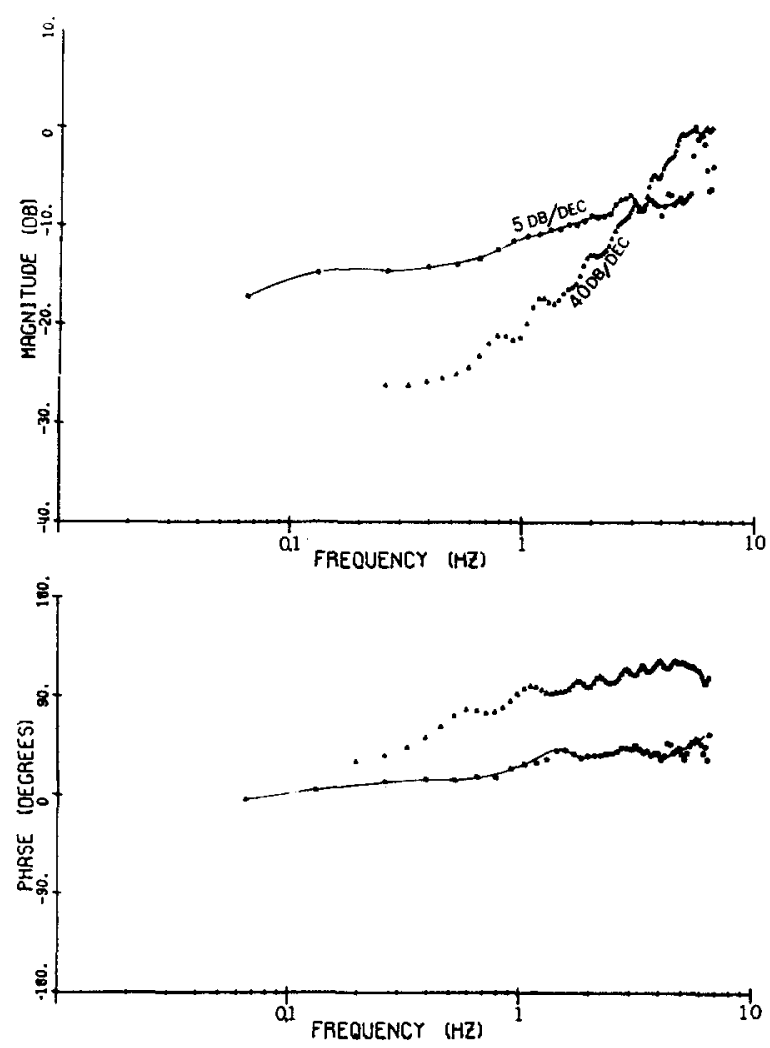

Fig. 7. Comparison of the frequency response profiles of a slowly adapting receptor at the knee joint level and a typical gracile cell. The slope of the Bode plot magnitude curve of the slowly adapting receptor is in the order of $5 \mathrm{~dB} /$ decade and the phase lead is a fraction of $90^{\circ}$. In contrast, the slope of the gracile magnitude response is about $40 \mathrm{~dB}$ /decade (above $1 \mathrm{~Hz}$ ) and the phase lead is greater than $90^{\circ}$. Slowly adapting response: solid line with symbols. Gracile response: symbols only.

cutaneous responses present at the gracile level is difficult. A satisfactory but more arduous way of resolving this problem would be to identify the gracile cells that respond to knee angle changes by direct stimulation without denervation of the posterior and medial articular nerves.

The effect of an anesthetic agent on the dynamic response of the secondary afferents must also be considered. Essentially, there are two questions that can be raised about our results. First, do the frequency profiles in these anesthetized preparations faithfully reflect the characteristics of the rapidly adapting afferent responses? Secondly, could anesthetic cause the absence of slowly adapting responses? Angel $^{2,3}$ has shown that the depressant effects of barbiturates on synaptic transmission in the cat somatosensory system is first manifested at the thalamic level. In addition, we have direct evidence that the first question can be answered affirmatively in the case of the type I responses. We have studied knee joint afferents in the ventrobasal complex (i.e., at the level of the third order cell) of completely unanesthetized cats and have found rapidly adapting cells with response profiles very similar 


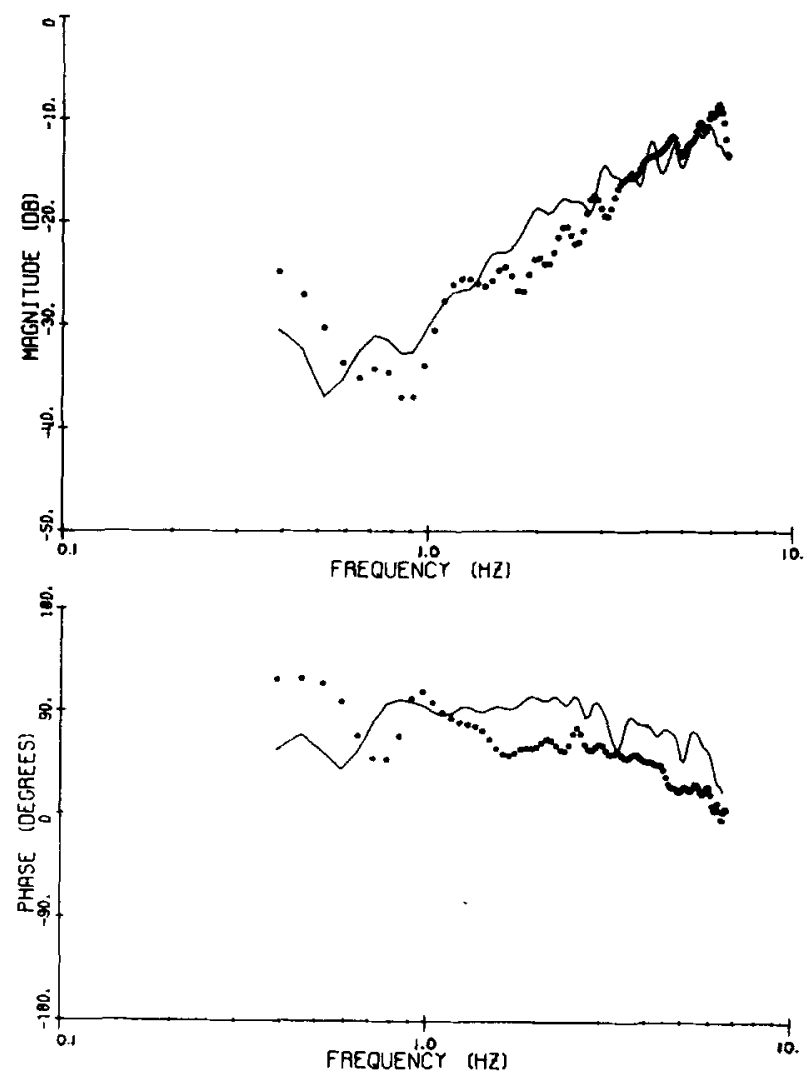

Fig. 8. Comparison of frequency response profiles obtained under anesthetized and unanesthetized conditions. Recording at the thalamic level. Filled circles, unanesthetized; solid line, anesthetized. These were type I responses. The $40 \mathrm{~dB} /$ decade characteristic slope of the magnitude portion of the Bode plot is present in both cases. The two response curves were obtained with two different animals. These results indicate that the dynamic characteristics of the type I responses are not altered significantly by anesthesia at normal levels.

to those of the type I cells ${ }^{44}$. Fig. 8 compares the Bode plots of the type I response at the thalamic level in a barbiturate cat with the response in an unanesthetized cat under similar stimulation.

The second question is more difficult to resolve. Indirect evidence suggests that the anesthetic level used in our experiments was not responsible for the absence of tonic joint responses at the gracile level. On numerous occasions tonically responding cells were found and usually traced to a cutaneous receptive field beyond the denervated area. Since the tonic cutaneous responses were not abolished by the anesthetic, one would find it necessary to attribute to the anesthetic a modality specific effect that selectively abolishes or alters tonic joint responses, but not tonic cutaneous responses at the gracile level. While this may be possible we do not think it is likely. Furthermore, the reports of Burgess and Clark suggest that the slowly adapting afferents from the hind limbs do not reach the gracile nucleus.

It is possible that the anesthetic effect may convert the tonic responses ob- 
served at the receptor level into the type II responses observed at the gracile level. In this case the apparent velocity sensitivity would be due to an anesthetic effect and not a true velocity sensitivity. Because of the indirect evidence cited in the previous paragraph we do not think this is the case. Further evidence that this is not the case is based on recent results at the receptor level suggesting that receptors exist at the primary level with response characteristics similar to the type II responses observed at the gracile level.

This question could be directly resolved by one of two approaches. The preparation could be converted to an unanesthetized, decerebrate preparation, or an awake animal could be prepared and studied in a manner similar to that used in our thalamic level studies. The absence or presence of tonic joint responses at the gracile level could then be more clearly interpreted. The unanesthetized gracile preparation seems to be beyond technical capabilities at present. The skull and several of the vertebrae would need to be fused in order to provide a stable recording situation since the recording site is near the junction of the skull and the vertebral column. The decerebrate preparation is certainly possible. The apparent small number of type II responses (only 5 in 36 animals) suggests that the chance of finding a cell that is truly a tonic responder under unanesthetized conditions and a velocity sensitive responder under anesthetic is very small. At the present time it does not seem to be warranted to perform a difficult series of experiments with such a low potential yield of information in light of present evidence. In due course further anatomically oriented studies may resolve the question of the projection or non-projection of tonic afferents from the knee joint to the gracile nucleus.

Although the existence of 'velocity sensitive' responses at the gracile level must be viewed with caution because of the possible anesthetic effects and the small number of such responses observed, the evidence for the existence of 'acceleration sensitive' responses seems compelling. The unanesthetized and anesthetized thalamic results are very similar. This precludes the possibility that the shape of these responses is an artifact of the anesthetic state. The $40 \mathrm{~dB} /$ decade slope indicative of acceleration sensitivity appears consistently in every frequency response profile obtained from type I cells. The details of bias angle sensitivity and excursion sensitivity may be subject to some anesthetic bias, but not the basic $40 \mathrm{~dB} /$ decade slope of the frequency response profile. Thus the evidence for acceleration sensitivity is very strong. This does not mean that the CNS uses the information in this form, but merely that there are peripheral mechanisms for generation and transmission of acceleration information.

\section{Effects of joint loading}

At best an experimental preparation is only an approximation of the normal condition. The experimental compromises made in this study with regard to stimulus application should be given some attention. The loading of the joint under the experimental condition is not normal from several aspects. As previously mentioned the denervation procedure changes the normal muscle tone and the associated joint loading. Results obtained in cases where the denervation procedure was not carried 
out are qualitatively quite similar to the results obtained in the denervated preparations, however. The joint loading under normal conditions includes a component of the weight of the cat. This component is absent in the experimental case.

The joint may be articulated in several angular directions in the normal course of events. We have attempted to impose a one-dimensional angulation stimulus in our experimental design. The joint is constrained to move in a particular articulation profile by the mechanical fixture. Although these considerations and their possible effects on experimental results must be kept in mind, we do not feel that the principal dynamic features of the responses reported in this study are subject to serious modification under a wide range of joint loadings and angulation profiles. This is based on extensive observation of the response characteristics of the joint receptors under a variety of experimental conditions.

\section{Modeling}

At the level of the nucleus gracilis the most common response to knee joint movement appears to be acceleration sensitivity (see Table I). This response can be characterized as the response of a non-linear accelerometer with even harmonic distortion characteristics. Hence, results obtained from the application of system identification techniques such as those employed in this study must be interpreted with care. In general, it is not possible to use a transfer function obtained from a nonlinear system to predict the response of the system to other input signals, whereas if the system were linear such predictions could be made. Nevertheless, the frequency response profile as a function of bias angle and excursion, the harmonic content of the output, and the step response of the system can provide insight into the nature of the signal processing properties of the system. The model fitted to our experimental data is not predictive in the linear sense; it does not predict the bi-directional character nor the harmonic content of the cellular responses. However the model does allow the sensitivity of the response at various bias angles and excursions to be assessed in terms of the gain $\mathrm{G}$. In addition the basic frequency response profile may be interpreted properly as that of an accelerometer in the range from 1 to $7 \mathrm{~Hz}$.

Since the system is complex, with definite non-linear characteristics, we feel that any particular form of systems formulation cannot be defended rigorously at this stage of investigation. However, we feel that our approach has provided valuable insight into many general features of joint receptor information coding and transmission. In addition it has been valuable in guiding our further experimentation.

\section{Comparisons}

A comparison of preliminary data obtained in our laboratory at the receptor and thalamic ${ }^{44}$ level with the data reported here at the gracile level reveals a similar acceleration sensitive response at all 3 levels. The bi-directional character and even harmonic non-linearity of the response appears to be most pronounced at the receptor level and least pronounced at the thalamic level ${ }^{44}$. The character of the fundamental (first harmonic) response at the 3 levels is similar. Responses similar to the type I responses observed at the gracile level are under study at the receptor level as well. 
Based on these observations we feel that the nervous system may selectively relay first harmonic information and that a describing function approach based on the first harmonic component is of value in investigating this system. The more detailed features of the higher harmonic components and the change in their relative contributions from level-to-level is of considerable interest and will be examined later.

Some investigators have found a rostral-caudal differentiation in the gracile nucleus with deep neurons located in the more rostral portions. We have not found this to be so. If anything, more joint cells were found caudal to the obex. Kruger et al. ${ }^{24}$ also found no evidence of modality segregation at any level. Our results agree with these findings and support the somatotopic mapping of the caudal segments of the body to the medial aspects of the nucleus gracilis.

Given the relatively large number of myelinated fibers in the articular nerve ( 176 approximately in the posterior nerve and 145 in the medial nerve ${ }^{36}$ ) it may seem a bit surprising that cells responding to joint motion are so rare in the gracile nucleus. However, only about $25 \%$ of the fibers that enter the dorsal columns reach the cervical levels ${ }^{20}$. In addition, Burgess and Clark found only an average of 20 first order fibers from both the medial and posterior nerves that projected as far as $C_{2}$. Thus, considering this small number of first order afferents, it is not surprising that it is so difficult to find cells responding to joint motion at the gracile level.

\section{Functional role}

The functional role of the types of joint responses found at the gracile level is open to question. Wall's hypothesis ${ }^{38,39}$ that signals carried via the dorsal columns serve to control a message search on other channels does not conflict with our findings. The acceleration sensitive cells are often exquisitely sensitive to the slightest joint motion. They could serve well as motion alarm signals. Possibly then the CNS would be prompted to direct (gate) attention to position sensitive responses. However, occurrences of both acceleration and possible velocity sensitivity in gracile cells lead us to suggest that they may also have a role in the sensation of dynamic limb motion as well. The functional significance of these gracile cell responses could be tested by the proper psychophysical experiments. For example, section of only the dorsal columns would be expected to have a minimal effect on static limb position sensation but appropriate experiments might demonstrate that there is a loss of velocity and acceleration information.

\section{APPENDIX}

The relation between the Bode plot magnitude and the mathematical operation of differentiation of a signal is presented here. Assume a signal of the form $x(t)=A$ $\sin (\omega t)$. If this signal is passed through a transducer with pure gain $G$, the output of the transducer will be of the form $y(t)=$ GA $\sin (\omega t)$. Thus $y$ is of constant amplitude for all frequencies. Such would be the case for a 'pure' position transducer.

If the transducer simply differentiates the input $x(t)$, then $y(t)=A \omega \cos (\omega t)$. Differentiation of position yields velocity. One can observe that the magnitude of $\mathrm{y}(\mathrm{t})$ is a function of $\mathrm{A} \omega$ in this case. From the formal definition of the decibel, 
$\mathrm{y}_{\mathrm{dB}}=20 \log _{10}(\mathrm{~A} \omega)$, or $\mathrm{y}_{\mathrm{dB}}=20 \log _{10}(\mathrm{~A})+20 \log _{10}(\omega)$. The slope of a plot of $\mathrm{y}_{\mathrm{dB}}$ versus $\log _{10}(\omega)$ is $20 \mathrm{~dB} /$ decade, and conversely a slope of $20 \mathrm{~dB} /$ decade implies differentiation. Any transducer that differentiates position, $x(t)$, may be termed 'velocity sensitive'.

If the transducer differentiates $x(t)$ twice, acceleration is described since the derivative of velocity is acceleration. In this case $y(t)=-\mathbf{A} \omega^{2} \sin (\omega t)$. The magnitude of $y(t)$ in decibels is:

$$
\begin{aligned}
\mathrm{y}_{\mathrm{dB}} & =20 \log _{10}\left(\mathrm{~A} \omega^{2}\right) \\
& =20 \log _{10}(\mathrm{~A})+20 \log _{10}\left(\omega^{2}\right) \\
& =20 \log _{10}(\mathrm{~A})+40 \log _{10}(\omega)
\end{aligned}
$$

Here the slope of the $y_{d B}$ versus the $\log _{10}(\omega)$ on the magnitude portion of the Bode plot is $40 \mathrm{~dB} /$ decade. Therefore a $40 \mathrm{~dB} /$ decade slope implies acceleration sensitivity.

A Bode magnitude plot with a constant slope of less than $20 \mathrm{~dB} /$ decade implies fractional order differentiation. For example, a slope of $5 \mathrm{~dB} /$ decade implies a 'quarter-order differentiator', and a transfer function of the form $\mathrm{G} \cdot \mathrm{s} \cdot \mathbf{2 5}$. This is a legitimate generalized operator and probably implies a system with distributed rather than 'lumped' parameters.

\section{ACKNOWLEDGEMENTS}

This work was supported by U.S. Public Health Service Grant NS 08470.

\section{REFERENCES}

1 ANDREw, B. L., AND DODT, E., The deployment of sensory endings at the knee joint of the cat, Acta physiol. scand.; 28 (1953) 287-296.

2 ANGel, A., Cortical responses to paired stimuli applied peripherally and at sites along the somatosensory pathway, J. Physiol. (Lond.), 191 (1967) 427-448.

3 ANGEL, A., The central control of sensory transmission and its possible relation to reaction time, Acta psychol. (Amst.), 30 (1969) 339-357.

4 Boyd, I. A., And Roberts, T. D. M., Proprioceptive discharges from the stretch receptors in the knee joint of the cat, J. Physiol. (I ond.), 122 (1953) 38-58.

5 Brown, M. C., AND STEIN, R. B., Quantitative studies on the slowly adapting stretch receptor of the crayfish, Kybernetik, 3 (1966) 175-185.

6 Browne, K., LeE, J., ANd Ring, P. A., The sensation of passive movement at the metatarsophalangeal joint of the great toe in man, J. Physiol. (Lond.), 126 (1954) 448-458.

7 Burgess, P. R., AND Clark, F. J., Dorsal column projection of fibers from the cat knee joint, J. Physiol. (Lond.), 203 (1969) 301-315.

8 Burgess, P. R., AND Clark, F. J., Characteristics of knee joint receptors in the cat, J. Physiol. (Lond.), 203 (1969) 317-335.

9 Chapman, K. M., AND Smith, R. S., A linear transfer function underlying impulse frequency modulation in a cockroach mechanoreceptor, Nature (Lond.), 197 (1963) 699-701.

10 Clark, F. J., Central projection of sensory fibers from the cat knee joint, J. Neurobiol., 3 (1972) 101-110.

11 Cohen, L. A., Activity of knee joint proprioceptors recorded from the posterior articular nerve, Yale J. Biol. Med., 28 (1955) 225-232.

12 CoOK, A. W., AND Browder, E. J., Function of posterior columns in man, Arch. Neurol. Psychiat. (Chic.), 12 (1965) 72-79.

13 Eklund, G., ANd Skoglund, S., On the specificity of the Ruffini-like joint receptors, Acta physiol. scand., 49 (1960) 184-191.

14 Freeman, M. A. R., And Wyke, B. D., The innervation of the knee joint. An anatomical and histological study in the cat, J. Anat. (Lond.), 101 (1967) 505-532. 
15 Gardner, E., The distribution and termination of nerves in the knee joint of the cat, $J$. comp. Neurol., 80 (1944) 11-33.

16 Gardner, E., Conduction rates and dorsal root inflow of sensory fibers from the knee joint of the cat, Amer. J. Physiol., 152 (1948) 436-446.

17 Gardner, E., Pathways to the cerebral cortex for nerve impulses from joints, Acta anat. (Basel), 73, Suppl. 56 (1969) 203-216.

18 Gesink, J. W., AND Williams, W. J., A servo controlled linear actuator for bio-system studies, Proc. 21st A.C.E.M.B., 10 (1968) 18.5.

19 Gilman, S., AND DENny-Brown, D., Disorders of movement and behavior following dorsal column lesions, Brain, 89 (1966) 397-418.

20 Glees, P., AND Soler, J., Fibre content of the posterior columns and synaptic connections of nucleus gracilis, $Z$. Zellforsch., 36 (1951) 381-400.

21 Goldscheider, A., Physiologie des Muskelsinnes, Barth, Leipzig, 1898.

22 KIRKwood, P. A., The frequency response of frog muscle spindles under various conditions, J. Physiol. (Lond.), 222 (1972) 135-160.

23 Korner, L., and LandGren, S., Projections of low threshold joint afferents to the cerebral cortex of the cat, Acta physiol. scand., 76 (1969) 5A-7A.

24 Kruger, L., Siminoff, R., And Witkovsky, P., Single neuron analysis of dorsal column nuclei of the cat, $J$. Neurophysiol., 24 (1961) 333-349.

25 Matthews, P. B. C., AND Stein, R. B., The sensitivity of muscle spindle afferents to small sinusoidal changes of length, J. Physiol. (Lond.), 200 (1969) 723-743.

26 MCCall, W. D., JR., Dynamic Response of Slowly Adapting Cat Knee Joint Receptors, Ph. D. Thesis, Univ. Michigan, Ann Arbor, 1971.

27 MCCall, W. D., JR., AND Williams, W. J., Dynamic analysis of cat knee joint receptors, Fed. Proc., 30 (1971) 709.

28 Merton, P. A., Human position sense and sense of effort, Symp. Soc. exp. Biol., 18 (1964) 387-400.

29 Milhorn, H. T., The Application of Control Theory to Physiological Systems, Saunders, Philadelphia, 1966, pp. 167-193.

30 Mountcastle, V. B., Modality and topographic properties of single neurons of cat's somatic sensory cortex, J. Neurophysiol., 20 (1957) 408-434.

31 Mountcastle, V. B., Poggio, G. F., and Werner, G., The relation of thalamic cell response to peripheral stimuli varied over an intensive continuum, J. Neurophysiol., 26 (1963) 807-834.

32 Norrsell, U., and Voorhoeve, P., Tactile pathways from the hind limb to the cerebral cortex in cat, Acta physiol. scand., 54 (1962) 9-17.

33 Poggio, G. F., and Mountcastle, V. B., The functional properties of ventrobasal thalamic neurons studied in unanesthetized monkeys, J. Neurophysiol., 26 (1963) 775-806.

34 Poppele, R. E., AND Bowman, R., Quantitative description of linear behavior of mammalian muscle spindles, J. Neurophysiol, 33 (1970) 59-72.

35 Provins, K. A., The effect of peripheral nerve block on the appreciation and execution of finger movements, J. Physiol. (Lond.), 143 (1958) 55-67.

36 Skoglund, S., Anatomical and physiological studies of knee joint innervation in the cat, Acta physiol. scand., 36, Suppl. 124 (1956) 1-101.

37 Vierck, C. J., Spinal pathways mediating limb position sense, Anat. Rec, 154 (1966) 437.

38 WALL, P. D., The sensory and motor role of impulses travelling in the dorsal columns towards cerebral cortex, Brain, 93 (1970) 505-524.

39 Wall, P. D., Sensory role of impulses travelling in the dorsal columns, IEEE Trans. ManMachine Syst., 11 (1970) 39-44.

40 Whitsel, B. L., Petrucelli, L. M., and Shapiro, G., Modality representation in the lumbar and cervical fasciculus gracilis of squirrel monkeys, Brain Research, 15 (1969) 67-78.

41 Williams, W. J., BeMent, S. L., Yin, T. C. T., and McCall, W. D., JR., Velocity and acceleration response in N. gracilis to angular joint motion, Soc. Neurosci. Abstr., First Ann. Meeting, Washington, D.C., 1971, Paper 10.8 .

42 Williams, W. J., Gesink, J. W., ANd Stern, M. M., Biological system transfer function extraction using swept-frequency and correlation technique, Med. biol. Engng, 10 (1972) 609-620.

43 Winter, D. L., N. gracilis of cat. Functional organization and corticofugal effects, J. Neurophysiol., 28 (1965) $48-70$.

44 YIN, T. C. T., AND Williams, W. J., Dynamic transfer characteristics of thalamic sensory neurons, Soc. Neurosci. Abstr., Second Ann. Meeting, Houston, Texas, 1972, Paper 36.3. 